\title{
Compact and Passive Parametric Macromodeling using Reference Macromodels and Positive Interpolation Operators
}

\author{
Francesco Ferranti, Member, IEEE, Tom Dhaene, Senior Member, IEEE, Luc Knockaert, Senior Member, IEEE
}

\begin{abstract}
We present an enhanced parametric macromodeling method that is able to generate compact and passive models over the entire design space of interest. It starts from a discrete set of data samples of the input-output system behavior (e.g. admittance, impedance and scattering parameters), which depend on multiple design variables such as layout and substrate parameters. The proposed approach generates accurate parametric macromodels whose size is not affected by the number of design parameters in addition to frequency. Stability and passivity are preserved over the design space of interest. Pertinent numerical results validate the proposed parametric macromodeling methods.
\end{abstract}

Index Terms-Parametric macromodeling, rational approximation, interpolation, passivity, compact models.

\section{INTRODUCTION}

During the design of large-scale digital or analog applications, it is important to predict the response of the system under study as a function of design parameters, such as geometrical and substrate characteristics. A typical design process includes design space optimization and exploration, and therefore it requires repeated simulations for different design parameter values. These design activities call for parametric macromodels that efficiently and accurately describe the complex behavior of such systems, which is typically characterized by the frequency (or time) and several design parameters. The accuracy, compactness and preservation of system properties (e.g. stability and passivity) are fundamental and required features of parametric macromodels.

Passivity-preserving parametric macromodeling techniques have been proposed in [1]-[6]. The passive interpolation of a set of stable and passive univariate macromodels, called root macromodels, treated as input-output systems has been proposed in [1], [2]. This interpolation process of inputoutput systems is robust, but it may not guarantee a high modeling capability. A passive interpolation of the statespace matrices of a set of root macromodels is proposed in [3]-[5], which provides an enhanced modeling capability with respect to [1], [2]. Unfortunately, these methods are sensitive to the issues related to the interpolation of statespace matrices [7] and the matrix solution of positive-real or

Manuscript received January 2012

Francesco Ferranti, Tom Dhaene and Luc Knockaert are with the Department of Information Technology, Internet Based Communication Networks and Services (IBCN), Ghent University - IBBT, Gaston Crommenlaan 8 Bus 201, B-9050 Gent, Belgium, email: \{francesco.ferranti, tom.dhaene,luc.knockaert\}@ugent.be. bounded-real lemma is used to perform a passivity-preserving interpolation of state-space matrices. Its computation can be carried out using Linear Matrix Inequalities (LMI) or Riccati equation solvers, which have a high computational cost [8]. A recent passivity-preserving parametric macromodeling method has been proposed in [6], which uses an innovative passive interpolation of root macromodels at an input-output level by means of a set of amplitude and frequency scaling coefficients to enhance the modeling capability of [1], [2]. The parametric macromodeling methods that use an input-output system level interpolation [1], [2], [6] are robust and accurate, but the order of the parametric macromodels may suffer from oversize due to the nature of the input-output system level interpolation. In these techniques, the design space is divided into cells and each cell is composed of vertex root macromodels which are combined to build a parametric macromodel at an inputoutput level. This input-output combination is responsible for the oversize.

This paper proposes an enhanced parametric macromodeling method that is able to generate compact and passive models over the entire design space of interest starting from a discrete set of parameterized data samples of the input-output system behavior (e.g. admittance (Y), impedance (Z) and scattering (S) parameters). First, the design space is divided into cells. Then, a passive reference macromodel is computed for each cell and it is used along with linear least-squares problems to model the cell vertices in a rational form. Amplitude scaling coefficients [6] are replaced and improved in this paper by means of linear least-square problems, which enhances the modeling capability. Finally, positive interpolation operators are used to parameterize the cell vertex models cell by cell. No additional input-output interpolation is required as in [1], [2], [6] and therefore the model order is not increasing with the number of design parameters leading to more compact models.

The paper is organized as follows. Some preliminaries are given in Section II. Section III describes the proposed parametric macromodeling approach. Section IV discusses the passivity-preserving feature of the presented method. Some considerations about the proposed approach and the model oversize effect are given in Section V. Some pertinent numerical results validate the proposed technique in Section VI. The conclusions are presented in Section VII.

\section{Preliminaries}

A parametric macromodel in the form 


$$
\mathbf{R}(s, \mathbf{g})=\mathbf{C}(\mathbf{g})(s \mathbf{I}-\mathbf{A}(\mathbf{g}))^{-1} \mathbf{B}(\mathbf{g})+\mathbf{D}(\mathbf{g})
$$

or equivalently

$$
\mathbf{R}(s, \mathbf{g})=\mathbf{C}_{0}(\mathbf{g})+\sum_{n=1}^{N(\mathbf{g})} \frac{\mathbf{C}_{n}(\mathbf{g})}{s-p_{n}(\mathbf{g})}
$$

is computed by the proposed parametric macromodeling method to accurately model a set of multivariate data samples $\left\{(s, \mathbf{g})_{k}, \mathbf{H}(s, \mathbf{g})_{k}\right\}_{k=1}^{K_{t o t}}$, which depend on the complex frequency $s=j \omega$ and $N$ design variables $\mathbf{g}=\left(g^{(n)}\right)_{n=1}^{N}$, such as layout features or substrate parameters. Two data grids are used in the modeling process: an estimation grid and a validation grid. The estimation grid is utilized to build a parametric macromodel. The validation grid is used to validate the modeling capability of the parametric macromodel in a set of points of the design space previously not used for its construction. The design space $\mathcal{D}(\mathbf{g})$ is considered as the parameter space $\mathcal{P}(s, \mathbf{g})$ without frequency. The parameter space $\mathcal{P}(s, \mathbf{g})$ contains all parameters $(s, \mathbf{g})$. If the parameter space is $(\mathrm{N}+1)$-dimensional, the design space is $\mathrm{N}$-dimensional [6]. The estimation design space grid is divided into cells using hyperrectangles (regular grids) [9] or simplices (regular and scattered grids) [10]. We note that the proposed parametric macromodeling method can handle regular and scattered estimation design space grids. The validation design space points are located in the center and centroid of each cell of the regular and scattered estimation grids. The cell of a N-D regular and scattered grid are N-D hyperrectangles and N-D simplices, respectively.

\section{PARAMETRIC MACROMODELING BASED ON REFERENCE MACROMODELS}

\section{A. Reference macromodels and frequency scaling coefficients}

We indicate a cell region of the design space as $\Omega_{i}, i=$ $1, \ldots, P$ and the corresponding vertices as $\mathbf{g}_{k}^{\Omega_{i}}, k=1, \ldots, Q$. For each cell a reference macromodel $\boldsymbol{R}_{r e f}\left(s, \Omega_{i}\right)$ is computed based on a set of frequency-domain data samples selected as reference data. The reference data used to build the reference macromodel for each cell is chosen among the cell data vertices $\mathbf{H}\left(s, \mathbf{g}_{k}^{\Omega_{i}}\right)$. For a vertex cell $\mathbf{g}_{k}^{\Omega_{i}}$, a corresponding reference macromodel is built, then it is parameterized and validated. If it is accurate with respect to an error threshold, the modeling of a cell is complete, otherwise the other cell vertices become candidate to be the reference macromodel to be parameterized. A parameterized reference macromodel must be accurate in the corresponding cell, therefore its accuracy is checked in all vertices and the validation point of its cell.

When a vertex cell $\mathbf{g}_{k}^{\Omega_{i}}$ is selected, the Vector Fitting (VF) method [11] can be used to identify the reference macromodel in the following from

$$
\mathbf{R}_{r e f}\left(s, \Omega_{i}\right)=\mathbf{C}_{0}\left(\mathbf{g}_{k}^{\Omega_{i}}\right)+\sum_{n=1}^{N\left(\Omega_{i}\right)} \frac{\mathbf{C}_{n}\left(\mathbf{g}_{k}^{\Omega_{i}}\right)}{s-p_{r e f, n}\left(\Omega_{i}\right)}
$$

starting from data samples $\mathbf{H}\left(s, \mathbf{g}_{k}^{\Omega_{i}}\right)$. A pole-flipping scheme is used to enforce stability [11], while passivity assessment and enforcement can be accomplished using the robust standard techniques [12], [13]. When the reference macromodel corresponding to one cell vertex is computed, the other vertex root macromodels $\mathbf{R}\left(s, \mathbf{g}_{k}^{\Omega_{i}}\right)$ of a specific cell are computed solving the optimization problem

$$
\min _{\alpha\left(\mathbf{g}_{j}^{\Omega_{i}}\right)} \operatorname{Err}\left(\widetilde{\boldsymbol{R}}_{r e f}\left(s, \mathbf{g}_{j}^{\Omega_{i}}\right), \mathbf{H}\left(s, \mathbf{g}_{j}^{\Omega_{i}}\right)\right), j \neq k
$$

with

$$
\begin{gathered}
\widetilde{\boldsymbol{R}}_{r e f}\left(s, \mathbf{g}_{j}^{\Omega_{i}}\right)=\boldsymbol{R}_{r e f}\left(s \alpha\left(\mathbf{g}_{j}^{\Omega_{i}}\right), \Omega_{i}\right) \\
\alpha\left(\mathbf{g}_{k}^{\Omega_{i}}\right)=1
\end{gathered}
$$

where $\alpha\left(\mathbf{g}_{j}^{\Omega_{i}}\right)$ are frequency scaling coefficients [6]. The optimization problem (4) can be solved using, for example, the Matlab [14] routines fmincon and fminsearchbnd with $\alpha\left(\mathbf{g}_{j}^{\Omega_{i}}\right)=1$ as initial guess. The frequency scaling coefficients must satisfy the following condition

$$
\alpha\left(\mathbf{g}_{j}^{\Omega_{i}}\right)>0
$$

to build overall passive parametric macromodels, as explained in Section IV. Once the frequency scaling coefficients are computed, linear least-square problems are solved to find suitable residues and direct terms such that

$$
\mathbf{R}\left(s, \mathbf{g}_{j}^{\Omega_{i}}\right) \simeq \mathbf{H}\left(s, \mathbf{g}_{j}^{\Omega_{i}}\right), j \neq k
$$

in a least-squares sense with

$$
\mathbf{R}\left(s, \mathbf{g}_{j}^{\Omega_{i}}\right)=\mathbf{C}_{0}\left(\mathbf{g}_{j}^{\Omega_{i}}\right)+\sum_{n=1}^{N\left(\Omega_{i}\right)} \frac{\mathbf{C}_{n}\left(\mathbf{g}_{j}^{\Omega_{i}}\right)}{s \alpha\left(\mathbf{g}_{j}^{\Omega_{i}}\right)-p_{r e f, n}\left(\Omega_{i}\right)}
$$

where $p_{r e f, n}\left(\Omega_{i}\right)$ are the poles of the reference macromodel $\boldsymbol{R}_{r e f}\left(s, \Omega_{i}\right)$. Passivity assessment and enforcement are performed on $\mathbf{R}\left(s, \mathbf{g}_{j}^{\Omega_{i}}\right)$. Each design space cell is described by its reference poles $p_{\text {ref, } n}\left(\Omega_{i}\right)$, vertex frequency scaling coefficients, residues and direct terms $\alpha\left(\mathbf{g}_{k}^{\Omega_{i}}\right), \mathbf{C}_{n}\left(\mathbf{g}_{k}^{\Omega_{i}}\right), \mathbf{C}_{0}\left(\mathbf{g}_{k}^{\Omega_{i}}\right)$, $k=1, \ldots, Q$.

The error function between two frequency responses $\mathbf{H}_{1}(s), \mathbf{H}_{2}(s)$ to be minimized in (4) can be adapted to the specific input-output representation under modeling. The following error can be used for scattering (S) parameters

$$
\begin{aligned}
& \operatorname{Err}\left(\mathbf{H}_{1}(s), \mathbf{H}_{2}(s)\right)= \\
& =\max _{i, j, k}\left(\left|H_{1,(i j)}\left(s_{k}\right)-H_{2,(i j)}\left(s_{k}\right)\right|\right)
\end{aligned}
$$

where $k=1, \ldots, K_{s}, i=1, \ldots, P_{\text {in }}, j=1, \ldots, P_{\text {out }}, K_{s}$, $P_{\text {in }}$ and $P_{\text {out }}$ are the number of frequency samples, input and output ports of the system, respectively. The RMS-error

$$
\begin{aligned}
& \operatorname{Err}\left(\mathbf{H}_{1}(s), \mathbf{H}_{2}(s)\right)= \\
& =\sqrt{\frac{\sum_{k=1}^{K_{s}} \sum_{i=1}^{P_{\text {in }}} \sum_{j=1}^{P_{\text {out }}}\left|H_{1,(i j)}\left(s_{k}\right)-H_{2,(i j)}\left(s_{k}\right)\right|^{2}}{P_{\text {in }} P_{\text {out }} K_{s}}}
\end{aligned}
$$


or weighted RMS-error

$$
\begin{aligned}
& \operatorname{Err}\left(\mathbf{H}_{1}(s), \mathbf{H}_{2}(s)\right)= \\
& =\sqrt{\frac{\sum_{k=1}^{K_{s}} \sum_{i=1}^{P_{\text {in }}} \sum_{j=1}^{P_{\text {out }}} \frac{\left|H_{1,(i j)}\left(s_{k}\right)-H_{2,(i j)}\left(s_{k}\right)\right|^{2}}{W_{(i j)}\left(s_{k}\right)}}{P_{\text {in }} P_{\text {out }} K_{s}}} \\
& W_{(i j)}\left(s_{k}\right)=\left|H_{2,(i j)}\left(s_{k}\right)\right|^{2}
\end{aligned}
$$

can be used for admittance $(\mathbf{Y})$ and impedance $(\mathbf{Z})$ parameters.

\section{B. Parameterization Step}

Once the vertex root macromodel $\mathbf{R}\left(s, \mathbf{g}_{k}^{\Omega_{i}}\right)$ are computed for a cell, the next step is building a parametric macromodel $\mathbf{R}(s, \mathbf{g})$ using suitable interpolation schemes. To describe the proposed algorithm more clearly, we consider a specific cell $\widehat{\Omega}$ composed of 4 data samples vertices in a 2-D design space $\mathbf{g}=\left(g^{(1)}, g^{(2)}\right)$ and a corresponding set of validation data samples, which is shown in Fig. 1. Fig. 2 summarizes the construction of the vertex root macromodel $\mathbf{R}\left(s, \mathbf{g}_{k}^{\Omega_{i}}\right)$.

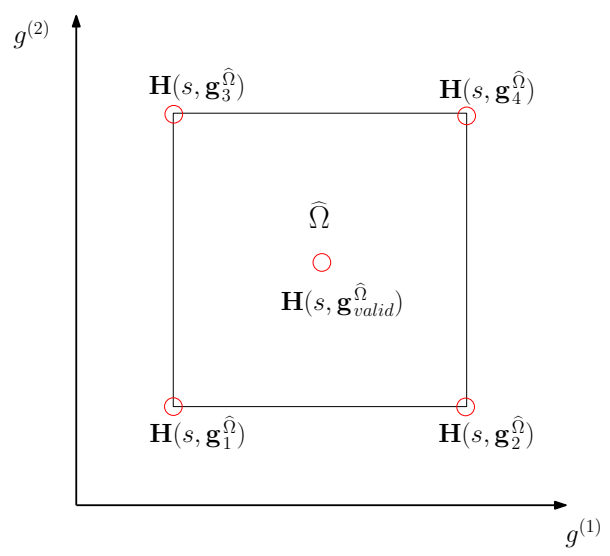

Fig. 1. Design space cell $\widehat{\Omega}$.

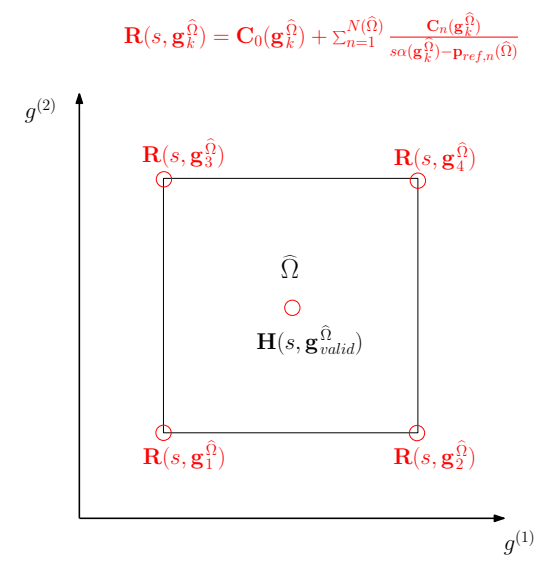

Fig. 2. Vertex root macromodels for the design space cell $\widehat{\Omega}$.

If the response of the system under modeling needs to be computed in a specific point of the design space cell $\widehat{\mathbf{g}} \widehat{\Omega}=$ $\left(\widehat{g}^{(1)}, \widehat{g}^{(2)}\right)^{\widehat{\Omega}}$, the vertex residues $\mathbf{C}_{n}\left(\mathbf{g}_{k}^{\widehat{\Omega}}\right)$, direct term $\mathbf{C}_{0}\left(\mathbf{g}_{k}^{\widehat{\Omega}}\right)$ and frequency scaling coefficients $\alpha\left(\mathbf{g}_{k}^{\widehat{\Omega}}\right)$ are interpolated in $\widehat{\mathbf{g}}^{\widehat{\Omega}}$ and a rational model

$$
\mathbf{R}\left(s, \widehat{\mathbf{g}}^{\widehat{\Omega}}\right)=\mathbf{C}_{0}\left(\widehat{\mathbf{g}}^{\widehat{\Omega}}\right)+\sum_{n=1}^{N(\widehat{\Omega})} \frac{\mathbf{C}_{n}\left(\widehat{\mathbf{g}}^{\widehat{\Omega}}\right)}{s \alpha\left(\widehat{\mathbf{g}}^{\widehat{\Omega}}\right)-\mathbf{p}_{\text {ref }, n}(\widehat{\Omega})}
$$

is obtained. Just as an example, let us use the piecewise multilinear method [9] for the interpolation, therefore we have for $k=1, \ldots, 4$ :

$$
\begin{aligned}
& \alpha\left(\widehat{\mathbf{g}}^{\widehat{\Omega}}\right)= \\
& =\sum_{k_{1}=1}^{2} \sum_{k_{2}=1}^{2} \alpha\left(\left(g_{k_{1}}^{(1)}, g_{k_{2}}^{(2)}\right)^{\widehat{\Omega}}\right) \ell_{k_{1}}\left(g^{(1)}\right) \ell_{k_{2}}\left(g^{(2)}\right) \quad \text { (15) } \\
& \mathbf{C}_{n}\left(\widehat{\mathbf{g}}^{\widehat{\Omega}}\right)= \\
& =\sum_{k_{1}=1}^{2} \sum_{k_{2}=1}^{2} \mathbf{C}_{n}\left(\left(g_{k_{1}}^{(1)}, g_{k_{2}}^{(2)}\right)^{\widehat{\Omega}}\right) \ell_{k_{1}}\left(g^{(1)}\right) \ell_{k_{2}}\left(g^{(2)}\right)(16) \\
& \mathbf{C}_{0}\left(\widehat{\mathbf{g}}^{\widehat{\Omega}}\right)= \\
& =\sum_{k_{1}=1}^{2} \sum_{k_{2}=1}^{2} \mathbf{C}_{0}\left(\left(g_{k_{1}}^{(1)}, g_{k_{2}}^{(2)}\right)^{\widehat{\Omega}}\right) \ell_{k_{1}}\left(g^{(1)}\right) \ell_{k_{2}}\left(g^{(2)}\right)(17)
\end{aligned}
$$

where each interpolation kernel function $\ell_{k_{j}}\left(g^{(j)}\right), j=1, \ldots, 2$ is selected as in piecewise linear interpolation.

The extension of the described procedure to the general multidimensional case is straightforward. We note that only scalar coefficients are optimized and linear least squares problems are solved. The interpolation process to obtain (14) does not lead to model oversize, since each cell is modeled by a constant number of poles and residues, and therefore the model order is not increasing with the number of design parameters due to input-output interpolation as it was the case in [1], [2], [6].

\section{Multivariate InTERPolation}

\section{A. Passivity Conditions}

When the macromodel is utilized in a circuit simulator for transient analysis, stability and passivity are fundamental properties to guarantee. It is known that, while a passive system is also stable, the reverse is not necessarily true. Passive systems cannot generate more energy than they absorb through their electrical ports. When the system is terminated on any arbitrary passive loads, none of them will cause the system to become unstable. A linear network described by admittance matrix $\mathbf{Y}(s)$ is passive if [15]:

1) $\mathbf{Y}\left(s^{*}\right)=\mathbf{Y}^{*}(s)$ for all $s$, where "*" is the complex conjugate operator.

2) $\mathbf{Y}(s)$ is analytic in $\Re e(s)>0$.

3) $\mathbf{Y}(s)$ is a positive-real matrix, i.e. : $\mathbf{z}^{* T}\left(\mathbf{Y}^{T}\left(s^{*}\right)+\mathbf{Y}(s)\right) \mathbf{z} \geq 0 ; \forall s: \Re e(s)>0$ and any arbitrary vector $\mathbf{z}$.

Similar results are valid for a linear network described by impedance matrix $\mathbf{Z}(s)$. 
The passivity of scattering input-output representations is also called nonexpansivity [16]. A linear network described by scattering matrix $\mathbf{S}(s)$ is passive if [15]:

1) $\mathbf{S}\left(s^{*}\right)=\mathbf{S}^{*}(s)$ for all $s$, where " $*$ " is the complex conjugate operator.

2) $\mathbf{S}(s)$ is analytic in $\Re e(s)>0$.

3) $\mathbf{I}-\mathbf{S}^{\mathrm{T}}\left(s^{*}\right) \mathbf{S}(s) \geq 0 ; \forall s: \Re e(s)>0$.

Condition 3 ) for nonexpansivity is equivalent to the condition $\|\mathbf{S}(s)\|_{\infty} \leq 1$ ( $\mathbf{H}_{\infty}$ norm) [16], i.e., the largest singular value of $\mathbf{S}(s)$ does not exceed one in the right-half $s$-plane.

Under the assumptions of having a minimal and strictly stable (all poles have a negative real part) state-space realization, an alternative and equivalent condition for the passivity of $\mathbf{Y}(s)$ and $\mathbf{S}(s)$ requires that the associated Hamiltonian matrix has no purely imaginary eigenvalues [16].

\section{B. Passivity Preserving Interpolation}

The parametrization step described in Section III-B uses positive interpolation operators [17] to parameterize frequency scaling coefficients, residues and directs terms. A rational model (14) can be seen as an interpolation of transfer functions with common poles.

A frequency scaling coefficient $\alpha$ is a compression or expansion term for the Laplace variable $s$. It is straightforward to prove that if $\alpha$ satisfies (7), passivity is preserved when it is applied to a passive system for $\mathbf{S}, \mathbf{Y}, \mathbf{Z}$ parameters. Positive interpolation operators guarantee (7) for $\alpha(\mathbf{g})$.

Once residues and direct terms are computed by solving linear least-squares problems to build vertex root macromodels, passivity is assessed end enforced. Since (14) can be seen as an interpolation of passive transfer functions with common poles, passivity over the entire design space of interest is guaranteed if the positive interpolation operators used to parameterize residues and direct terms satisfy some specific conditions [1], [2]. Interpolation kernel functions $\ell_{k_{j}}\left(g^{(j)}\right), j=1, \ldots, N$ must satisfy

$$
\begin{gathered}
\left.0 \leq \ell_{k_{j}}\left(g^{(j)}\right)\right) \leq 1 \\
\ell_{k_{j}}\left(g_{i}^{(j)}\right)=\delta_{k_{j}, i}
\end{gathered}
$$

for $\mathbf{Y}, \mathbf{Z}$ parameters and

$$
\begin{gathered}
\left.0 \leq \ell_{k_{j}}\left(g^{(j)}\right)\right) \leq 1, \\
\ell_{k_{j}}\left(g_{i}^{(j)}\right)=\delta_{k_{j}, i}, \\
\sum_{k_{j}=1}^{K_{j}} \ell_{k_{j}}\left(g^{(j)}\right)=1 .
\end{gathered}
$$

for $\mathbf{S}$ parameters. Interpolation methods that belong to the general class of positive interpolation operators can be used, e.g., the piecewise multilinear and multivariate simplicial methods [9]. We note that the interpolation kernel functions of these methods only depend on the design space grid points and their computation does not require the solution of a linear system to impose an interpolation constraint. Therefore, interpolating systems, matrices or scalars does not make any difference for the interpolation kernel functions.

\section{Discussion}

We summarize the main features of the new reference macromodel-based approach described in Section III:

- each design space cell is described by its reference poles, vertex frequency scaling coefficients, residues and direct terms;

- amplitude scaling coefficients [6] are replaced and improved by means of linear least-square problems, which are used to compute vertex residues and direct terms and enhance the modeling capability;

- the model oversize of [1], [2], [6] is avoided, since no input-output interpolation of systems is performed. The model order does not increase with the number of design parameters and model compactness is guaranteed even for a high number of design parameters;

- stability and passivity are guaranteed over the entire design space.

The state-space dimension of parametric macromodels is influenced by the fitting method (matrix-wise, column-wise or entry-wise) used for the reference macromodels and root macromodels and the interpolation schemes. Considering the model oversize in system interpolation-based methods [1], [2], [6], it is important to notice that if parametric macromodels are used in frequency-domain analyses, there is no need to realize them in a state-space form. We recall that the input-output interpolation process of state-space systems is responsible for the model oversize, since a weighted sum of linear timeinvariant (LTI) systems is equivalent to an LTI system with size equal to the sum of the sizes of the combined LTI systems. A pole-residue form is suitable for frequency-domain analyses and therefore the model oversize problem can be mitigated. Considering time-domain simulations that require a state-space form to integrate parametric macromodels with linear and nonlinear drivers and receivers, then the model oversize cannot be avoided and the new proposed method is really effective to solve it, without sacrificing accuracy. If the interpolation schemes use $Q$ vertices in the interpolation process, an average gain in the model order equal to $Q$ is achieved. E.g., $Q=2^{\mathrm{N}}$ and $Q=\mathrm{N}+1$ for the piecewise multilinear and simplicial interpolation methods [9], respectively. $\mathrm{N}$ represents the number of design parameters in addition to frequency. Therefore, the compactness guaranteed by the new proposed method becomes fundamental in high-dimensional problems.

\section{NUMERICAL EXAMPLES}

This section presents numerical results to validate the proposed parametric macromodeling method. The number of poles for reference macromodels and vertex root macromodels is selected adaptively in VF by a bottom-up approach, in such a way that the errors (10),(12) are smaller than $-50 \mathrm{~dB}$ and 0.05 , respectively. The number of poles to use as a starting point for the bottom-up approach is selected by computing the required number of poles to accurately model the corner data of the design space and choosing the minimum number in this set of poles. 
The worst case error of (10),(12) over the validation grid is chosen to assess the accuracy and quality of parametric macromodels:

$$
\begin{gathered}
\mathbf{g}_{\text {max }}=\underset{\mathbf{g} \in \text { validation grid }}{\operatorname{argmax}} \operatorname{Err}(\mathbf{R}(s, \mathbf{g}), \mathbf{H}(s, \mathbf{g})) \\
\operatorname{Err}=\operatorname{Err}\left(\mathbf{g}_{\text {max }}\right)
\end{gathered}
$$

We note that all the computations to build parametric macromodels performed by the proposed technique and [1], [2], [6] use a pole-residue form for the reference macromodels and root macromodels. All numerical experiments were implemented in Matlab R2009A [14] and carried out on Windows platform equipped with Intel Core2 Extreme CPU Q9300 $2.53 \mathrm{GHz}$ and $8 \mathrm{~GB}$ RAM.

\section{A. Three coupled microstrips}

Three coupled microstrips have been modeled in this example. The cross section is shown in Fig. 3. The conductors have width $w=100 \mu \mathrm{m}$ and thickness $t=50 \mu \mathrm{m}$, the spacing between the conductors is equal to $S=200 \mu \mathrm{m}$. The dielectric is $300 \mu \mathrm{m}$ thick and characterized by a dispersive and lossy permittivity which has been modeled by the wideband Debye model [18]. The per-unit-length parameters of this structure have been computed by the solver [19]. Then, we consider three parameters, namely the length $L$ of the lines and two fabrication parameters $\lambda_{1}, \lambda_{2}$ that influence the per-unit-length parameters

$$
\begin{aligned}
\mathbf{Z}_{\text {pul }}\left(s, \lambda_{1}\right) & =\left(1+\lambda_{1}\right) \mathbf{Z}_{\text {pul }}(s, 0) \\
\mathbf{Y}_{\text {pul }}\left(s, \lambda_{2}\right) & =\left(1+\lambda_{2}\right) \mathbf{Y}_{\text {pul }}(s, 0)
\end{aligned}
$$

Their corresponding ranges are shown in Table I.

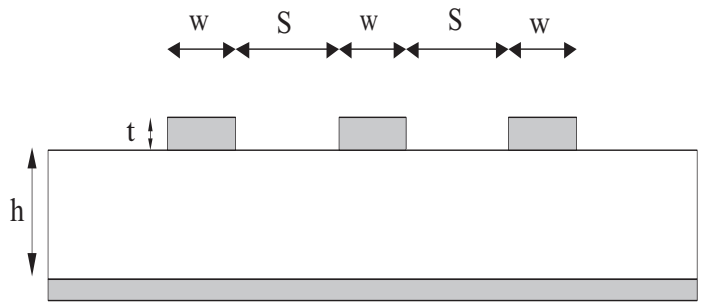

Fig. 3. Cross section of the three coupled microstrips.

TABLE I

DESIGN PARAMETERS OF THE THREE COUPLED MICROSTRIPS.

\begin{tabular}{|l|c|c|}
\hline Parameter & Min & Max \\
\hline Frequency (freq) & $100 \mathrm{kHz}$ & $15 \mathrm{GHz}$ \\
Length (L) & $1 \mathrm{~cm}$ & $1.3 \mathrm{~cm}$ \\
$\lambda_{1}$ & -0.15 & 0.15 \\
$\lambda_{2}$ & -0.15 & 0.15 \\
\hline
\end{tabular}

The scattering and admittance parameters $\mathbf{S}\left(s, L, \lambda_{1}, \lambda_{2}\right)$, $\mathbf{Y}\left(s, L, \lambda_{1}, \lambda_{2}\right)$ have been computed using the transmission line theory [20] for 251 frequency samples over an estimation grid of $5 \times 3 \times 3\left(L, \lambda_{1}, \lambda_{2}\right)$ samples and a validation grid of $4 \times 2 \times 2\left(L, \lambda_{1}, \lambda_{2}\right)$ samples. The new proposed approach and the technique presented in [6] are used to build 4-D macromodels. The multilinear interpolation scheme is used in the parameterization steps. First, we analyze the modeling of $\mathbf{S}$ parameters and then of $\mathbf{Y}$ parameters. Concerning $\mathbf{S}$ parameters, the order of the state-space realization (matrixwise fitting) of the reference macromodels is varying between 60 and 96 over the design space.

To clearly show the advantages of the new proposed method, it has been compared with the technique described in [6]. Table II shows some comparison measures: the error defined in (25) related to (10), the CPU time to build and validate the final passive parametric macromodels, and the maximum and minimum order of the parametric macromodels over the design space. In the CPU time to build and validate the final passive parametric macromodels, two contributions are identified: 1) the CPU time to build and validate the parametric macromodels and 2) the CPU time needed to assess and enforce passivity during the model construction.

TABLE II

COMPARISON FIRST EXAMPLE (S PARAMETERS),

\begin{tabular}{|l|l|l|l|}
\hline Method & Error (25) & CPU time & (Min,max) order \\
\hline Proposed & $-40.13 \mathrm{~dB}$ & $6 \mathrm{~min} 52 \mathrm{~s}+12 \min 14 \mathrm{~s}$ & $(66,90)$ \\
{$[6]$} & $-40.44 \mathrm{~dB}$ & $4 \min 38 \mathrm{~s}+6 \mathrm{~min} 47 \mathrm{~s}$ & $(60,690)$ \\
\hline
\end{tabular}

A gain in the model order of almost 8 is achieved with respect to [6] without sacrificing the accuracy. Some results obtained using the reference macromodel technique are shown in what follows. Fig. 4 shows the magnitude of the parametric macromodel of $\mathbf{S}_{11}\left(s, L, \lambda_{1}, \lambda_{2}\right)$ for $\lambda_{1}=0, \lambda_{2}=0$. Fig. 5 shows the magnitude of the parametric macromodel of $\mathbf{S}_{16}\left(s, L, \lambda_{1}, \lambda_{2}\right)$ for $L=1.15 \mathrm{~cm}, \lambda_{2}=0$. Fig. 6 compares $\mathbf{S}_{11}\left(s, L, \lambda_{1}, \lambda_{2}\right)$ and its macromodel for the values $L=1.1125 \mathrm{~cm}, \lambda_{1}=-0.075, \lambda_{2}=\{-0.075,0.075\}$ that have not been used in the estimation grid.

Concerning $\mathbf{Y}$ parameters, the order of the state-space realization (matrix-wise fitting) of the reference macromodels is varying between 78 and 126 over the design space. As previously, table III shows some comparison measures for the used parametric macromodeling methods. In this case, the error defined in (25) is related to (12).

TABLE III

COMPARISON FIRST EXAMPLE (Y PARAMETERS).

\begin{tabular}{|l|l|l|l|}
\hline Method & Error (25) & CPU time & $($ Min,max) order \\
\hline Proposed & 0.09 & $7 \min 25 \mathrm{~s}+10 \min 54 \mathrm{~s}$ & $(72,90)$ \\
{$[6]$} & 0.07 & $8 \min 37 \mathrm{~s}+7 \min 2 \mathrm{~s}$ & $(78,870)$ \\
\hline
\end{tabular}

Similar accuracy is achieved by the two techniques, while the new method obtains a gain in the model order of almost 10. In what follows, some results obtained using the reference macromodel technique are presented. Fig. 7 shows the 


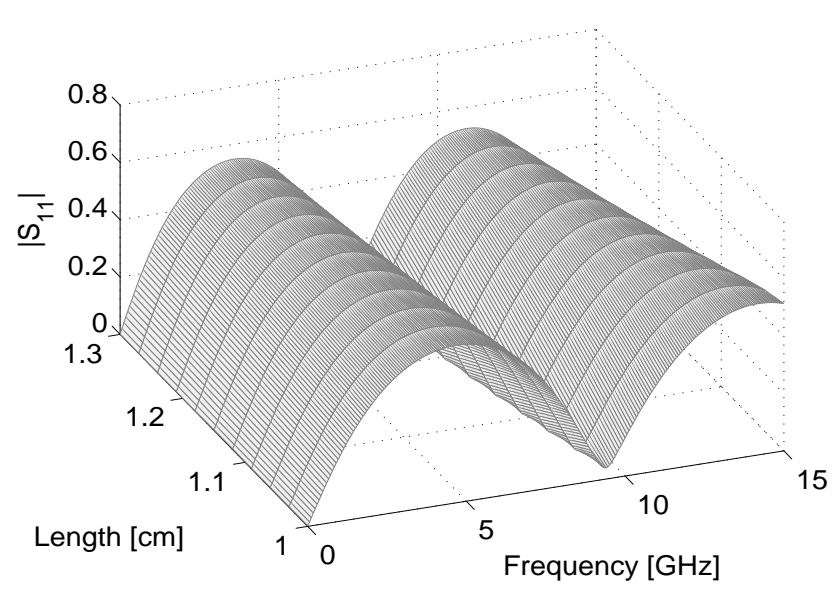

Fig. 4. Magnitude of the 4-D macromodel of $\mathbf{S}_{11}\left(s, L, \lambda_{1}, \lambda_{2}\right)\left(\lambda_{1}=\right.$ $0, \lambda_{2}=0$ ).

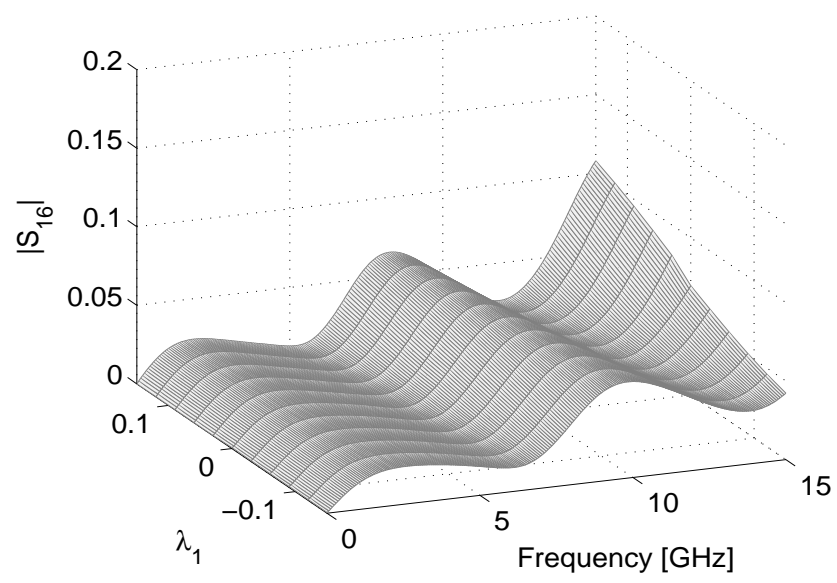

Fig. 5. Magnitude of the 4-D macromodel of $\mathbf{S}_{16}\left(s, L, \lambda_{1}, \lambda_{2}\right)(L=1.15$ $\left.\mathrm{cm}, \lambda_{2}=0\right)$.

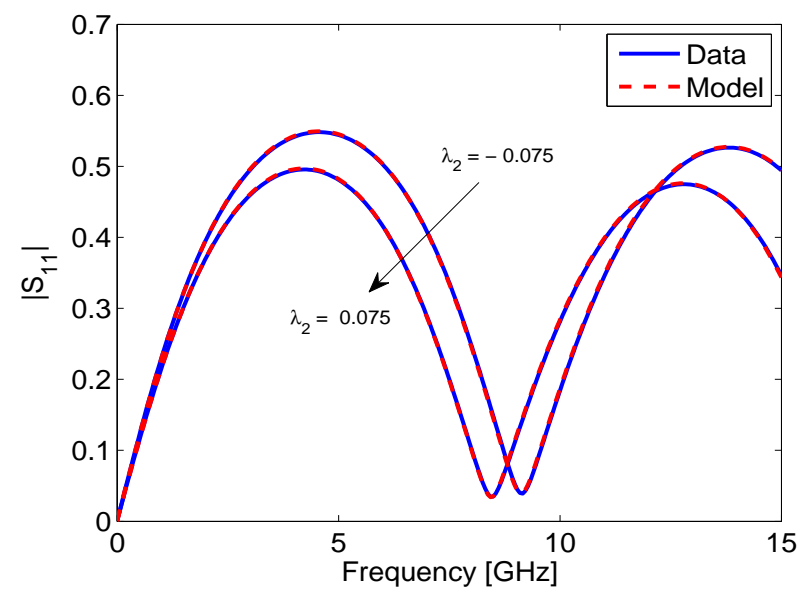

Fig. 6. Magnitude of the 4-D macromodel of $\mathbf{S}_{11}\left(s, L, \lambda_{1}, \lambda_{2}\right)(L=1.1125$ $\left.\mathrm{cm}, \lambda_{1}=-0.075, \lambda_{2}=\{-0.075,0.075\}\right)$.

magnitude of the parametric macromodel of $\mathbf{Y}_{12}\left(s, L, \lambda_{1}, \lambda_{2}\right)$ for $L=1.15 \mathrm{~cm}, \lambda_{2}=0$. Fig. 8 shows the magnitude of the parametric macromodel of $\mathbf{Y}_{11}\left(s, L, \lambda_{1}, \lambda_{2}\right)$ for $L=1.15 \mathrm{~cm}$, $\lambda_{1}=0$. Fig. 9 compares $\mathbf{Y}_{11}\left(s, L, \lambda_{1}, \lambda_{2}\right)$ and its macromodel for the values $L=\{1.0375,1.2625\} \mathrm{cm}, \lambda_{1}=-0.075$, $\lambda_{2}=-0.075$ which have not been used in the estimation grid. Fig. 10 shows the distribution of the minimum absolute value of the real part of Hamiltonian matrix eigenvalues over a dense sweep of the design space. Since there are no purely imaginary eigenvalues, the parametric macromodel is passive over the design space of interest [16]. Similar results can be obtain for the model of the $\mathbf{S}$ parameters.

The new proposed technique is able to build accurate and passive models over the entire design space of interest, which do not suffer from model oversize. The model order does not increase with the number of design parameters as in [6], since no input-output interpolation is performed. The method [6] achieves a similar accuracy with less compact models.

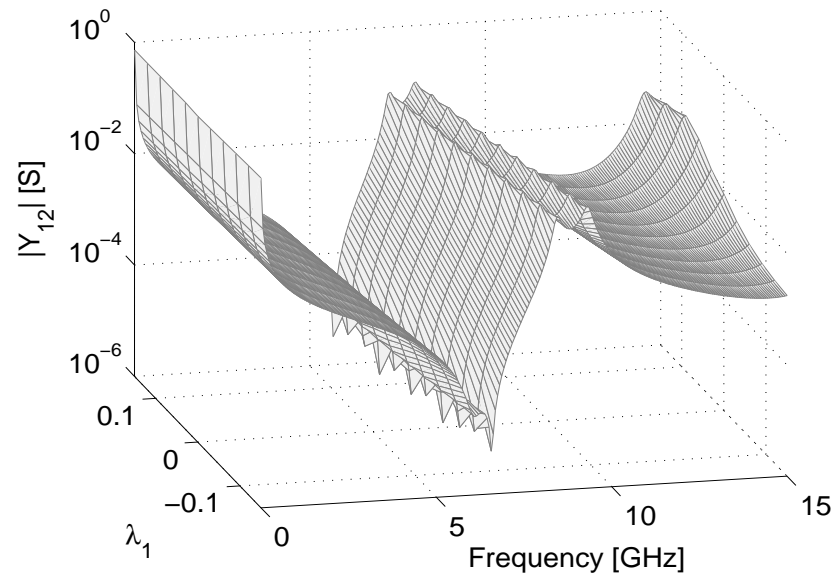

Fig. 7. Magnitude of the 4-D macromodel of $\mathbf{Y}_{12}\left(s, L, \lambda_{1}, \lambda_{2}\right)(L=1.15$ $\left.\mathrm{cm}, \lambda_{2}=0\right)$.

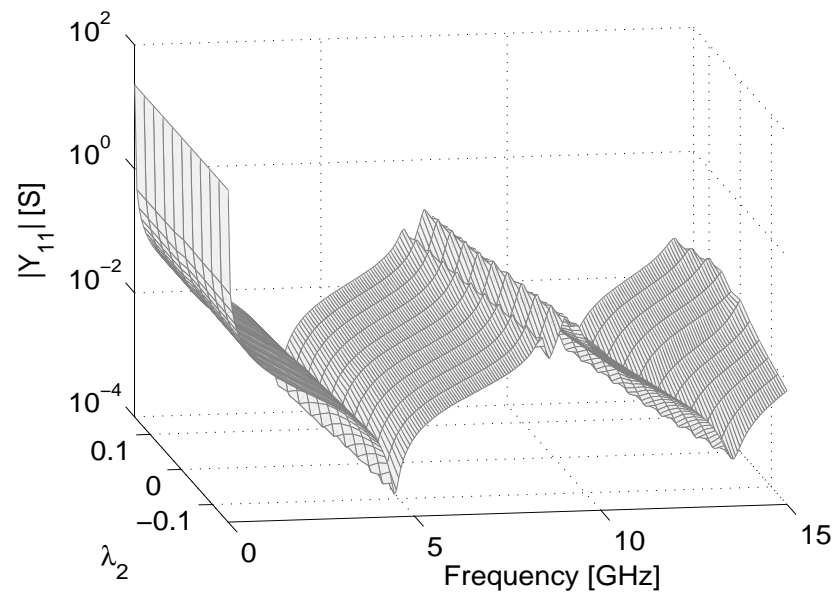

Fig. 8. Magnitude of the 4-D macromodel of $\mathbf{Y}_{11}\left(s, L, \lambda_{1}, \lambda_{2}\right)(L=1.15$ $\mathrm{cm}, \lambda_{1}=0$ ).

\section{B. Eight layers Printed Circuit Board}




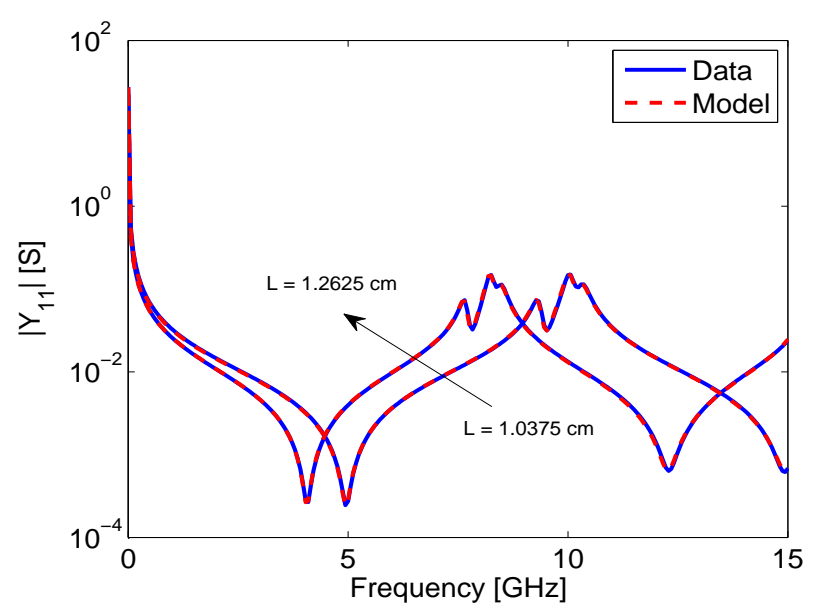

Fig. 9. Magnitude of the 4-D macromodel of $\mathbf{Y}_{11}\left(s, L, \lambda_{1}, \lambda_{2}\right)(L=$ $\left.\{1.0375,1.2625\} \mathrm{cm}, \lambda_{1}=-0.075, \lambda_{2}=-0.075\right)$.

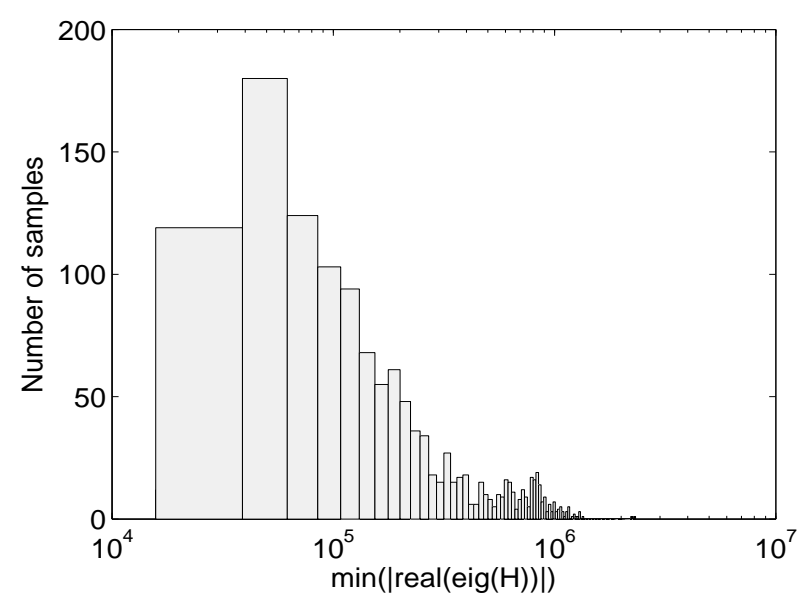

Fig. 10. Histogram: distribution of the minimum absolute value of the real part of Hamiltonian matrix eigenvalues.

A printed circuit board (PCB) with eight layers has been modeled in this example. The structure is shown in Fig. 11. The vias are connected to $3 \mathrm{~mm}$ long traces located on layer 6. The width of the traces is equal to $0.11 \mathrm{~mm}$ and the dielectric material of the substrate is FR4 with $\epsilon_{r}=3.63$ and loss tangent $\operatorname{tg}(\delta)=0.02$. A multipole Debye model is used to characterize the dieletric losses over the frequency range. We consider two design parameters in addition to frequency, namely the radius of the antipads $(R)$ and the center to center distance between signal vias and ground vias $(D)$. Their corresponding ranges are shown in Table IV.

TABLE IV

DESIGN PARAMETERS OF THE PCB.

\begin{tabular}{|l|c|c|}
\hline Parameter & Min & Max \\
\hline Frequency (freq) & $0 \mathrm{~Hz}$ & $20 \mathrm{GHz}$ \\
Antipads radius (R) & $0.4826 \mathrm{~mm}$ & $0.6026 \mathrm{~mm}$ \\
Distance (D) & $1.2525 \mathrm{~mm}$ & $2.4525 \mathrm{~mm}$ \\
\hline
\end{tabular}

The scattering parameters $\mathbf{S}(s, R, D)$ have been computed

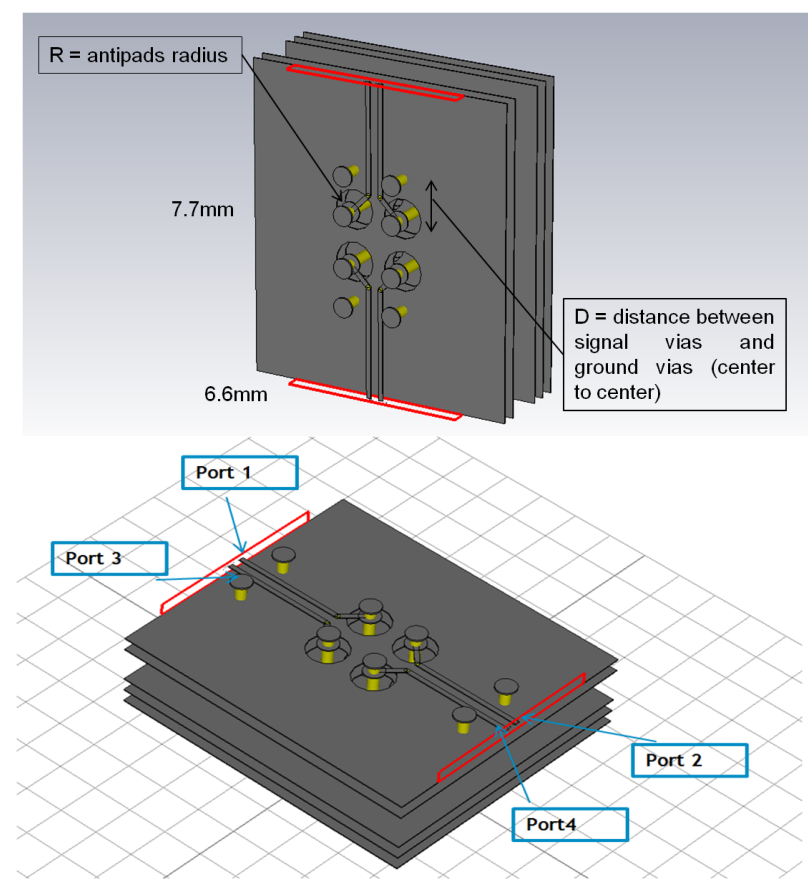

Fig. 11. PCB.

by means of the commercial software [21] for 1001 frequency samples over an estimation grid of $4 \times 6(R, D)$ samples and a validation grid of $3 \times 5(R, D)$ samples. The new proposed approach is used to build a 3-D macromodel. The multilinear interpolation scheme is used in the parameterization steps.

The order of the state-space realization (matrix-wise fitting) of the parametric macromodel is varying between 52 and 56 over the design space. The error defined in (25) related to (10) is equal to $-41 \mathrm{~dB}$.

Table V shows some comparison measures between the proposed method and the commercial solver used to obtain the data. In the CPU time to build the final passive parametric macromodel, two contributions are identified: 1) the CPU time to build the parametric macromodel and 2) the CPU time needed to assess and enforce passivity during the model construction.

TABLE V

COMPARISON SECOND EXAMPLE.

\begin{tabular}{|l|c|}
\hline Step & CPU time \\
\hline Estimation grid by solver $(4 \times 6)(\mathrm{R}, \mathrm{D})$ & $3 \mathrm{~h} 6 \mathrm{~min}$ \\
Validation grid by solver $(3 \times 5)(\mathrm{R}, \mathrm{D})$ & $1 \mathrm{~h} 56 \mathrm{~min} 15 \mathrm{~s}$ \\
Building model & $5 \mathrm{~min} 49 \mathrm{~s}+11 \mathrm{~min} 11 \mathrm{~s}$ \\
Validating model & $11 \mathrm{~s}$ \\
Evaluating solver (1001 frequency samples) & $7 \mathrm{~min} 45 \mathrm{~s}$ \\
Evaluating model (1001 frequency samples) & $0.1 \mathrm{~s}$ \\
\hline
\end{tabular}

Table V shows that:

1) the CPU time to build and validate the parametric macromodel is small with respect to the CPU time to gather the estimation and validation data samples;

2) the parametric macromodel is very efficient in evaluating the system response with respect to the used commercial 
solver. The parametric macromodel is able to achieve a speedup of 4650x for the computation of one frequency response. Therefore, performing design activities such as design space exploration, optimization, sensitivity analysis that require multiple simulations becomes very efficient using the proposed parametric macromodeling method.

Some results obtained using the reference macromodel technique are shown in what follows. Fig. 12 shows the magnitude of the parametric macromodel of the near-end crosstalk $\mathbf{S}_{31}(s, R, D)$ for $R=0.543 \mathrm{~mm}$. Fig. 13 compares $\mathbf{S}_{31}(s, R, D)$ and its macromodel for the values $R=0.543$ $\mathrm{mm}, D=\{1.3725,1.8525,2.3325\} \mathrm{mm}$ that have not been used in the estimation grid.

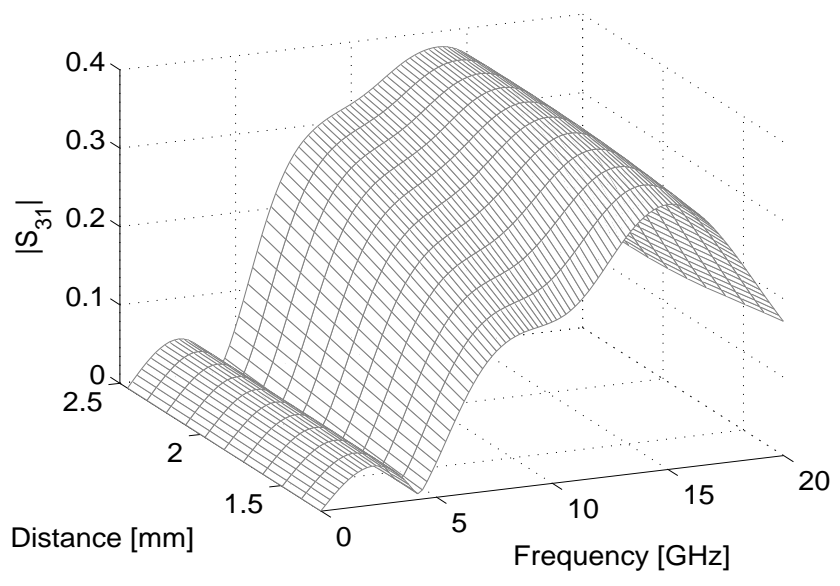

Fig. 12. Magnitude of the 3-D macromodel of $\mathbf{S}_{31}(s, R, D)(R=0.543$ $\mathrm{mm})$.

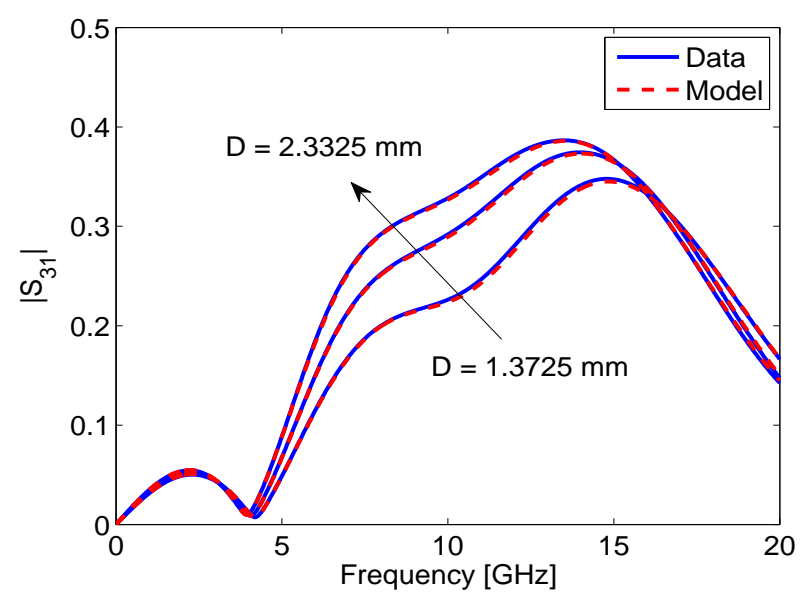

Fig. 13. Magnitude of the 3-D macromodel of $\mathbf{S}_{31}(s, R, D)(R=0.543$ $\mathrm{mm}, D=\{1.3725,1.8525,2.3325\} \mathrm{mm})$.

Fig. 14 shows the distribution of the minimum absolute value of the real part of Hamiltonian matrix eigenvalues over a dense sweep of the design space. As in the previous example, the parametric macromodel is passive over the design space of interest.

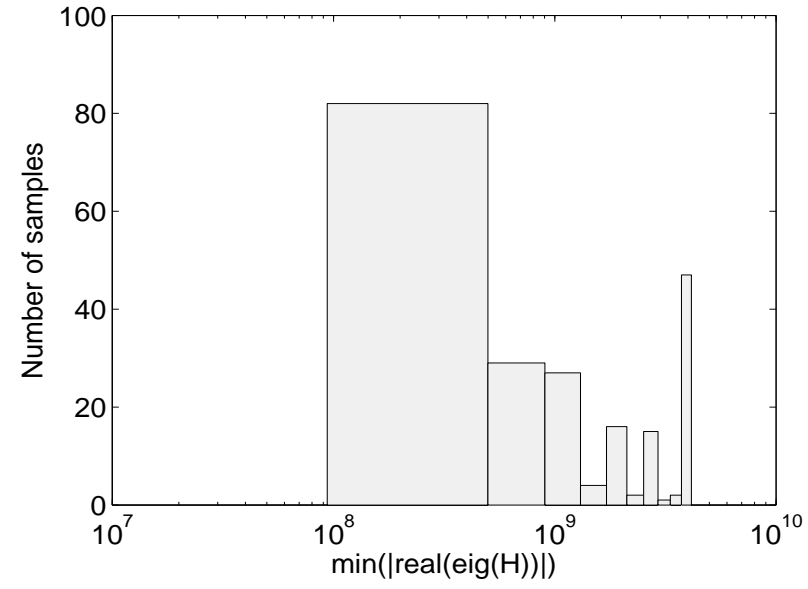

Fig. 14. Histogram: distribution of the minimum absolute value of the real part of Hamiltonian matrix eigenvalues.

\section{CONCLUSIONS}

We have presented an enhanced parametric macromodeling scheme that is able to generate compact and passive macromodels over the entire design space of interest starting from a discrete set of parameterized data samples of the input-output system behavior (e.g. admittance, impedance and scattering parameters). The model oversize of system interpolation-based methods due to input-output system interpolation is avoided in the new technique, while stability and passivity are guaranteed over the design space. Numerical simulations confirm the high modeling capability of the proposed method and the compactness of the generated models.

\section{REFERENCES}

[1] F. Ferranti, L. Knockaert, and T. Dhaene, "Guaranteed passive parameterized admittance-based macromodeling," IEEE Trans. Adv. Packag., vol. 33, no. 3, pp. $623-629$, Aug. 2010

[2] _ _ "Parameterized S-parameter based macromodeling with guaranteed passivity," IEEE Microw. Wireless Compon. Lett., vol. 19, no. 10, pp. 608-610, Oct. 2009.

[3] F. Ferranti, L. Knockaert, T. Dhaene, and G. Antonini, "Passivitypreserving parametric macromodeling for highly dynamic tabulated data based on Lur'e equations," IEEE Trans. Microw. Theory Tech., vol. 58, no. 12, pp. 3688-3696, Dec. 2010.

[4] P. Triverio, M. Nakhla, and S. Grivet-Talocia, "Passive parametric macromodeling from sampled frequency data," in IEEE 14th Workshop on Signal Propagation on Interconnects (SPI), 2010, May 2010, pp. 117 $-120$.

[5] — - "Passive parametric modeling of interconnects and packaging components from sampled impedance, admittance or scattering data," in Electronic System-Integration Technology Conference (ESTC), 2010, Sept. 2010, pp. 1-6.

[6] F. Ferranti, L. Knockaert, and T. Dhaene, "Passivity-preserving parametric macromodeling by means of scaled and shifted state-space systems," IEEE Trans. Microw. Theory Tech., vol. 59, no. 10, pp. 2394-2403, Oct. 2011.

[7] J. De Caigny, J. F. Camino, and J. Swevers, "Interpolating model identification for SISO linear parameter-varying systems," Mechanical Systems and Signal Processing, vol. 23, no. 8, pp. 2395 - 2417, 2009.

[8] L. Knockaert, T. Dhaene, F. Ferranti, and D. De Zutter, "Model order reduction with preservation of passivity, non-expansivity and markov moments," Systems \& Control Letters, vol. 60, no. 1, pp. 53-61, Jan. 2011.

[9] W. A. Weiser and S. E. Zarantonello, "A note on piecewise linear and multilinear table interpolation in many dimensions," Mathematics of Computation, vol. 50, no. 181, pp. 253-264, Jan. 1988. 
[10] D. F. Watson, "Computing the n-dimensional Delaunay tessellation with application to Voronoi polytopes," The Computer Journal, vol. 24, no. 2, pp. 167-172, Febr. 1981.

[11] B. Gustavsen and A. Semlyen, "Rational approximation of frequency domain responses by vector fitting," IEEE Trans. Power Delivery, vol. 14, no. 3, pp. 1052-1061, Jul. 1999.

[12] — , "A half-size singularity test matrix for fast and reliable passivity assessment of rational models," IEEE Trans. Power Delivery, vol. 24, no. 1, pp. 345-351, Jan. 2009.

[13] B. Gustavsen, "Fast passivity enforcement for S-parameter models by perturbation of residue matrix eigenvalues," IEEE Transactions on Advanced Packaging, vol. 33, no. 1, pp. 257-265, Feb. 2010.

[14] "MATLAB R2009A User's Guide,", www.mathworks.com.

[15] B. D. Anderson, S. Vongpanitlerd, Network Analysis and Synthesis. Englewood Cliffs, NJ: Prentice-Hall, 1973.

[16] S. Boyd, L. El Ghaoui, E. Feron and V. Balakrishnan, Linear Matrix Inequalities in System and Control Theory. Philadelphia, PA: SIAM, 1994, vol. 15.

[17] G. Allasia, "A class of interpolating positive linear operators: Theoretical and computational aspects," in Recent Developments in Approximation Theory, Wavelets and Applications, S. P. Singh, Ed. Dordrecht: Kluwer, 1995, pp. 1-36.

[18] A.R.Djordjević, R.M. Biljić, V.D. Likar-Smiljanić, T.K. Sarkar, "Wideband frequency-domain characterization of FR-4 and time-domain causality," IEEE Transactions on Electromagnetic Compatibility, vol. 43, no. 4, pp. 662-667, Nov. 2001.

[19] Simbeor, Electromagnetic Simulation Environment with 3D Full-Wave Field Solver for Multi-Layered Circuits, Simberian Inc., Seattle.

[20] C. R. Paul, Analysis of Multiconductor Transmission Lines, 2nd ed. New York, NY: John Wiley \& Sons, 2008.

[21] CST STUDIO SUITE 2012, www.cst.com.

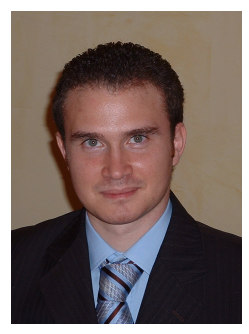

Francesco Ferranti (M'10) received the B.S. degree (summa cum laude) in electronic engineering from the Università degli Studi di Palermo, Palermo, Italy, in 2005, the M.S. degree (summa cum laude and honors) in electronic engineering from the Università degli Studi dell'Aquila, L'Aquila, Italy, in 2007, and the Ph.D. degree in electrical engineering from the University of Ghent, Ghent, Belgium, in 2011. He is currently a Post-Doctoral Research Fellow with the Department of Information Technology (INTEC), Ghent University, Ghent, Belgium. His research interests include parametric macromodeling, parameterized model order reduction, electromagnetic compatibility and signal integrity numerical modeling, stochastic modeling, system identification.

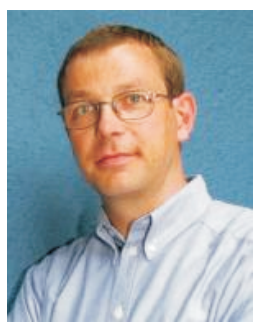

Tom Dhaene (SM'05) was born in Deinze, Belgium, on June 25, 1966. He received the Ph.D. degree in electrotechnical engineering from the University of Ghent, Ghent, Belgium, in 1993. From 1989 to 1993, he was Research Assistant at the University of Ghent, in the Department of Information Technology, where his research focused on different aspects of full-wave electro-magnetic circuit modeling, transient simulation, and time-domain characterization of high-frequency and high-speed interconnections. In 1993, he joined the EDA company Alphabit (now part of Agilent). He was one of the key developers of the planar EM simulator ADS Momentum. Since September 2000, he has been a Professor in the Department of Mathematics and Computer Science at the University of Antwerp, Antwerp, Belgium. Since October 2007, he is a Full Professor in the Department of Information Technology (INTEC) at Ghent University, Ghent, Belgium. As author or co-author, he has contributed to more than 150 peer-reviewed papers and abstracts in international conference proceedings, journals and books. He is the holder of 3 US patents.

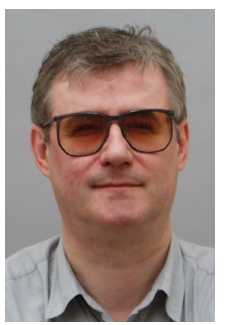

Luc Knockaert (SM'00) received the M. Sc. Degree in physical engineering, the $\mathrm{M}$. Sc. Degree in telecommunications engineering and the $\mathrm{Ph}$. D. Degree in electrical engineering from Ghent University, Belgium, in 1974, 1977 and 1987, respectively. From 1979 to 1984 and from 1988 to 1995 he was working in North-South cooperation and development projects at the Universities of the Democratic Republic of the Congo and Burundi. $\mathrm{He}$ is presently affiliated with the Interdisciplinary Institute for BroadBand Technologies (www.ibbt.be) and a professor at the Dept. of Information Technology, Ghent University (www.intec.ugent.be). His current interests are the application of linear algebra and adaptive methods in signal estimation, model order reduction and computational electromagnetics. As author or co-author he has contributed to more than 100 international journal and conference publications. He is a member of MAA, SIAM and a senior member of IEEE. 